\title{
Life-stage factors associated with overweight severity in adolescents
}

\author{
Raziye Dut $^{1 \oplus}$, Antonio Videira-Silva ${ }^{2 \oplus}$, Ana Sofia Vilardouro ${ }^{3 \oplus}$, Silvia Freira ${ }^{3 \odot}$, \\ Helena Fonseca ${ }^{4,5}$ \\ ${ }^{1}$ Department of Pediatrics, İstanbul Education and Research Hospital, İstanbul, Turkey; ${ }^{2}$ Pediatric University Clinic, Faculty of \\ Medicine, University of Lisbon, Portugal, ${ }^{4,5}$ Division of Adolescent Medicine, ${ }^{3}$ Department of Pediatrics, Hospital de Santa Maria, \\ Lisboa, Portugal.
}

\begin{abstract}
Background. Investigating life-stage factors associated with overweight may be useful in the prevention of excessive BMI increase. The main aim of this study was to investigate the influence of the route of delivery, birth weight and overweight onset on overweight severity in a sample of overweight adolescents followed at a Pediatric Obesity Clinic.
\end{abstract}

Methods. Clinical data from 412 adolescents with overweight (BMI $\geq$ p85), aged 10-18 were retrospectively collected and analyzed.

Results. Adolescents born by cesarean section (CS) showed a lower age of overweight onset, compared to other methods of delivery $(d=0.33, p=.009)$. Birth weight was positively associated with BMI z-score $(r=.164, p=$ 002) and waist circumference (WC) $(r=.191, p=.001)$. The overweight onset was negatively associated with BMI $z$-score $(r=-.277, p<.001)$, WC $(r=-.270, p<.001)$ and body fat mass $(r=-.199, p=.001)$. Overweight duration was the best predictor of BMI $z$-score, explaining in $75 \%$ its variation $(\mathrm{F}=1,317)=26.94, p<.001)$, which increased to $99 \%$ when birth weight was included in the model $(\mathrm{F}(2,316)=18.47, p<.001)$.

Conclusions. This study suggests that lifestyle may interrupt the burden of CS on BMI z-score throughout growth. Moreover, increased birth weight may anticipate overweight onset, and consequently overweight duration in the presence of inadequate lifestyle behaviors.

Key words: adolescent, birth weight, way of delivery, overweight, overweight severity.

Obesity is a major chronic disease and public health concern around the world. Over a third of the world population has obesity. ${ }^{1}$ A secular trend of the increasing prevalence of obesity has been estimated with $38 \%$ of the world's adult population developing overweight and another $20 \%$ obesity, by $2030 .^{2}$

Besides the way of delivery, with an acknowledged association between cesarean section (CS) and obesity, the three critical periods for the development of childhood

凶 Antonio Videira-Silva

antonioascenso@campus.ul.pt

Received 25th November 2019, revised 5th January 2020, accepted 18th February 2020. obesity include fetal life, the period of adiposity rebound (4-6 years old), and adolescence..$^{3-5}$

According to the developmental origins of health and disease hypothesis, adverse conditions throughout early development, either in the uterus or during the early postnatal years or both, may lead to metabolic changes that increase obesity risk later on. ${ }^{6}$ In that line, the prenatal period is referred to as a 'critical period' where adverse events may have a lifelong effect on body composition and contribute to the development of obesity. ${ }^{7}$ Based on the International Study of Childhood Obesity, Lifestyle and the Environment (ISCOLE) which covered urban and sub-urban regions in twelve countries, high birth weight (defined as birth weight $\geq 3500 \mathrm{~g}$ ) was associated with increased 
odds of obesity. ${ }^{8}$ Gillman et al. ${ }^{9}$ showed that high birth weight was a risk for overweight in adolescence. Other studies have shown that birth weight is linear and proportionally correlated to body mass index (BMI) $\cdot{ }^{10}$ Frisancho et al. ${ }^{11}$ showed that the relative risk for high BMI in adolescence was 1.9-times higher for children who were born as small for gestational age (SGA), 2.2-times for appropriate weight for gestational age (AGA), and 5.7-fold for large for gestational age (LGA). On the other hand, other authors have reported a relationship between SGA and adulthood obesity. ${ }^{12}$ The supporting evidence explaining these results is based on the "J-shaped" (association of low birth weight with increased body fat mass (BFM)) as well as on the "U-shaped" curve hypothesis (association of both low and high birth weight with BFM). ${ }^{13}$

The lack of consensus suggests further more complex associations between birth weight and obesity. The period of adiposity rebound corresponds to the second rise in the BMI curve occurring between the ages of 4 and 6 , when the $\mathrm{BMI}$ begins to increase again, after a rise in infancy and a subsequent decline. ${ }^{14}$ This is another critical period for the development of childhood obesity that can track to early adulthood. ${ }^{15}$ According to Geserick et al. $^{16}$ children who become overweight during this period have higher odds of being obese at adolescence. Adolescence may be considered as the last critical period for the development of obesity. ${ }^{17}$

Although the prevalence of obesity has come to a plateau in many European countries, the severity of obesity seems to increase, especially among adolescents. ${ }^{18}$ Due to the increased obesity severity and its related comorbidities, several authors have suggested that waist circumference (WC) and waist-height ratio (WHtR) should be routinely assessed and used as measures of central adiposity. ${ }^{19,20}$

It is crucial to identify early life-stage factors associated with overweight in order to prevent excessive BMI increase till reaching adulthood. In fact, it has been shown that adolescent obesity is associated with increased risk of obesity during adulthood with $70.5 \%$ of severely obese adolescents remaining obese as adults. ${ }^{21}$

Although several studies have analyzed the relationship between some perinatal factors, such as the way of delivery, birth weight or overweight onset and BMI in children, to the best of our knowledge, the interaction between these factors in adolescents with overweight has never been investigated so far.

The main aim of this study was to investigate the influence of the way of delivery, birth weight for gestational age and overweight onset on adolescent overweight severity. We have hypothesized that: (i) CS is a predictor of increased BMI and waist circumference during adolescence; (ii) birth weight for gestational age is positively associated with BMI and WC during adolescence; (iii) overweight onset during the period of adiposity rebound is a predictor of BMI and WC severity during adolescence.

\section{Material and Methods}

\section{Participants}

Clinical files from adolescents with overweight (BMI $\geq$ p85), aged 10-18, with a first appointment between October 2014 and December 2018 at the Pediatric Obesity Clinic, Hospital de Santa Maria, Lisbon, Portugal, were searched and data collected retrospectively.

This study was approved by the research ethics committee of the Faculty of Medicine of the University of Lisbon, Portugal (271/2016), and is in accordance with the 1964 Helsinki declaration and its later amendments or comparable ethical standards. Signed informed assent/consent was signed by all the participants and respective caregivers.

\section{Measures and instruments}

Birth weight, gestational age and way of delivery

Birth weight, gestational age and way of delivery were collected using the individual health 
booklet, and birth weight for gestational age was categorized according to INTERGROWTH 21st. ${ }^{22}$

Birth weights ranging from percentile 11 to 89 for gestational age were considered as AGA, at percentile 10 or under as SGA and at percentile 90 or above as LGA. The z-score of birth weight for gestational age was also computed according to INTERGROWTH 21st. ${ }^{22}$

\section{Overweight onset and overweight duration}

Overweight onset, considered as the time where BMI started to exceeded percentile 85 based on the World Health Organization charts, was assessed using the individual health booklet.

Overweight duration was calculated as the difference between current age and overweight onset. Both variables were registered in years.

\section{Anthropometric and body composition assessment}

Height was assessed with a height stadiometer (SECA 217, Hamburg, Germany) in the Frankfurt plan, without shoes, with the participants back to the stadiometer, and after an expiratory phase. Height was registered to the nearest $0.1 \mathrm{~cm}$.

Bodyweight and body composition were measured with a bioelectrical impedance scale (InBody 230, Seoul, Korea) to the nearest 0.1 $\mathrm{kg}$, with the subjects wearing as few clothes as possible, and without shoes or socks. \% of BFM and \% of skeletal muscle mass (SMM) were calculated dividing the total BFM and SMM $(\mathrm{kg})$ by body weight, respectively.

BMI was calculated dividing the body weight in kilograms by the square of height in meters [BMI= weight $(\mathrm{kg}) /$ height $\left.^{2}(\mathrm{~m})\right]$. The BMI z-score was calculated according to World Health Organization [BMI z-score $=[(\mathrm{BMI} / \mathrm{M}(t))$ $\mathrm{L}(\mathrm{t})-1] / \mathrm{L}(t) S(t)]$.

WC was assessed using a flexible anthropometric tape (SECA 203, Hamburg, Germany). WC was measured at the iliac crest level, with the subjects standing and at the end of a regular expiration (Cameron method).
WHtR was calculated dividing the WC in centimeters by the height in centimeters [WHtR $=W C(\mathrm{~cm}) /$ Height $(\mathrm{cm})]$.

\section{Clinical assessments}

Pubertal status was assessed and categorized according to Tanner stages.

\section{Statistical analysis}

Data was analyzed using the IBM SPSS statistics (IBM SPSS statistics, version 21.0, IBM, New York, USA).

Chi-square and Independent sample t-test were used in order to analyze gender differences. Because statistically significant differences between girls and boys were found, all the analyses were performed controlling for sex.

The associations between gestational age, birth weight, overweight onset, and all the anthropometric and body composition variables were analyzed using partial correlations, controlling for age, sex and way of delivery. BMI z-score prediction (dependent variable) was computed using multiple linear regressions (stepwise method). A $p$ value of $<.05$ was considered statistically significant.

\section{Results}

Clinical data from 412 overweight adolescents (87.1\% Caucasian) were analyzed.

Boys ( $n=192$, mean age $13.9 \pm 2.0)$ showed higher height $(d=0.53, p<.001)$, BMI z-score $(d=$ $0.21, p=.040)$, WC $(d=0.21, p=.048)$ and SMM $(d=$ $0.40, p<.001)$, compared to girls $(n=220$, mean age $14.4 \pm 2.2$ ). On the other hand, girls showed higher BFM $(d=0.53, p<.001)$ compared to boys (Table I).

Statistically significant differences between girls and boys were also found in the number of CS, with a higher number of CS among boys (46.6\% vs. $29.5 \%, p=.001$ ) (Table I). Adolescents born by CS showed a lower age of overweight onset, compared to those born with other methods of delivery $(d=0.33, p=.009)$. 
Table I. Sample characteristics.

\begin{tabular}{|c|c|c|c|c|c|c|c|}
\hline & \multicolumn{2}{|c|}{ Girls } & \multicolumn{2}{|c|}{ Boys } & \multirow[b]{2}{*}{$p$} & \multicolumn{2}{|c|}{ Total } \\
\hline & $n$ & Mean $\pm S D$ & $n$ & Mean $\pm S D$ & & $n$ & Mean $\pm S D$ \\
\hline Age (years) & 220 & $14.4 \pm 2.2$ & 192 & $13.9 \pm 2.0$ & .017 & 412 & $14.2 \pm 2.1$ \\
\hline Height (cm) & 220 & $158.9 \pm 7.5$ & 192 & $163.7 \pm 10.6$ & $<.001$ & 412 & $161.1 \pm 9.4$ \\
\hline Weight (kg) & 220 & $82.9 \pm 18.6$ & 192 & $86.5 \pm 24.2$ & .098 & 412 & $84.5 \pm 21.5$ \\
\hline BMI $\left(\mathrm{kg} / \mathrm{m}^{2}\right)$ & 220 & $32.59 \pm 6.02$ & 192 & $31.78 \pm 6.36$ & .186 & 412 & $32.21 \pm 6.19$ \\
\hline BMI z-score & 220 & $2.68 \pm 1.02$ & 192 & $2.89 \pm 0.99$ & .040 & 412 & $2.78 \pm 1.01$ \\
\hline $\mathrm{WC}(\mathrm{cm})$ & 189 & $101.5 \pm 12.9$ & 152 & $104.4 \pm 14.5$ & .048 & 341 & $102.8 \pm 13.7$ \\
\hline WHtR & 189 & $0.63 \pm 0.07$ & 152 & $0.63 \pm 0.07$ & .681 & 341 & $0.63 \pm 0.07$ \\
\hline BFM (\%) & 193 & $44.1 \pm 5.7$ & 180 & $40.7 \pm 7.2$ & $<.001$ & 373 & $42.5 \pm 6.7$ \\
\hline SMM (\%) & 175 & $31.8 \pm 6.1$ & 158 & $34.5 \pm 7.2$ & $<.001$ & 333 & $33.1 \pm 6.8$ \\
\hline Overweight onset (years) & 163 & $6.2 \pm 4.3$ & 161 & $5.3 \pm 3.65$ & .042 & 324 & $5.7 \pm 4.03$ \\
\hline Birth weight (g) & 214 & $3261 \pm 554$ & 191 & $3287 \pm 659$ & .664 & 405 & $3273 \pm 605$ \\
\hline Gestational age (weeks) & 220 & $38.8 \pm 1.8$ & 192 & $38.1 \pm 2.6$ & .001 & 412 & $38.4 \pm 2.2$ \\
\hline Birth weight z-score & 214 & $0.40 \pm 1.29$ & 191 & $0.51 \pm 1.24$ & .365 & 405 & $0.45 \pm 1.27$ \\
\hline Race & $n$ & $\%$ & $n$ & $\%$ & $p$ & $n$ & $\%$ \\
\hline Caucasian & 192 & 87.3 & 167 & 87.0 & & 359 & 87.1 \\
\hline Black & 27 & 12.3 & 24 & 12.5 & $.993^{*}$ & 51 & 12.4 \\
\hline Asian & 1 & 0.5 & 1 & 0.5 & & 2 & 0.5 \\
\hline \multicolumn{8}{|l|}{ Delivery } \\
\hline Eutocic & 114 & 60.0 & 84 & 47.2 & & 198 & 53.8 \\
\hline Cesarian section & 56 & 29.5 & 83 & 46.6 & $.001^{*}$ & 139 & 37.8 \\
\hline Other & 20 & 10.5 & 11 & 6.2 & & 31 & 8.4 \\
\hline \multicolumn{8}{|l|}{ Birth weight category } \\
\hline SGA & 28 & 13.1 & 9 & 4.7 & & 37 & 9.1 \\
\hline AGA & 131 & 61.2 & 137 & 71.7 & $.008^{*}$ & 268 & 66.2 \\
\hline LGA & 55 & 25.7 & 45 & 23.4 & & 100 & 24.7 \\
\hline \multicolumn{8}{|l|}{ Tanners' stage } \\
\hline 1 & 1 & 12.5 & 7 & 87.5 & & 8 & 2.2 \\
\hline 2 & 25 & 31.6 & 54 & 68.4 & & 79 & 21.4 \\
\hline 3 & 18 & 39.1 & 28 & 60.9 & $<.001^{*}$ & 46 & 12.4 \\
\hline 4 & 29 & 43.3 & 38 & 56.7 & & 67 & 18.1 \\
\hline 5 & 124 & 72.9 & 46 & 27.1 & & 170 & 45.9 \\
\hline
\end{tabular}

AGA: appropriate weight for gestational age, BFM: body fat mass, BMI: body mass index, CS: cesarean section, LGA: large for gestational age, SGA: small for gestational age, SMM: skeletal muscle mass, WC: waist circumference, WHtR: waist-toheight ratio.

* Chi-square test.

From the 412 adolescents, 37 were born SGA and 100 LGA. According to birth weight for gestational age, LGA participants showed a higher BMI z-score during adolescence compared to AGA ( $d=0.32, p=.023)$. No statistically significant differences were found between SGA and AGA or between SGA and LGA.
No associations were found between birth weight for gestational age and overweight onset.

When controlling for sex, age and way of delivery, birth weight showed to be positively correlated with BMI z-score $(r=.164, p=.002)$, WC $(r=.191, p=.001)$ and WHtR $(r=.126, p=.028)$; birth 
weight $\mathrm{z}$-score was positively correlated with BMI z-score $(r=.144, p=.006)$ and WC $(r=.153$, $p=.007) ;$ overweight onset was negatively correlated with BMI z-score $(r=-.277, p<.001)$, WC $(r=-.270, p<.001)$, WHtR $(r=-.227, p<.001)$, and BFM ( $r=-.199, p=.001)$; overweight duration showed to be positively correlated with BMI z-score $(r=-.261, p<.001)$, WC $(r=-.260, p$ $<.001)$, WHtR $(r=-.213, p<.001)$, and BFM ( $r=$ -.176, $p=.003)$. Gestational age was positively correlated with birth weight $(r=.472, p<.001)$, and negatively correlated with birth weight $\mathrm{z}$-score $(r=-.234, p<.001)$ (Table II).

According to multiple linear regressions (stepwise method), overweight duration was the best predictor of BMI z-score during adolescence, explaining in $75 \%$ its variation $(\mathrm{F}(1,317)=26.94, p<.001)$. Including birth weight in the model, the interaction between these variables was able to explain $99 \%$ of BMI z-score variation $(\mathrm{F}(2,316)=18.47, p<.001)$.

Overweight onset during the period of adiposity rebound was not associated with BMI and WC severity.

\section{Discussion}

Adolescent overweight dramatically progresses into adulthood. It is crucial to investigate lifestage factors associated with overweight in order to prevent excessive BMI increase throughout this critical period for the development of obesity. ${ }^{17,23,24}$

The main aim of this study was to investigate the influence of perinatal factors (i.e. way of delivery, birth weight) and overweight onset on overweight severity in a sample of overweight adolescents followed at a Pediatric Obesity Clinic, in order to identify the factors associated with BMI severity in this population. Identifying these factors may allow the improvement of both preventive and timely weight management interventions.

Literature shows a strong association between CS and increased BMI and WC, in the offspring

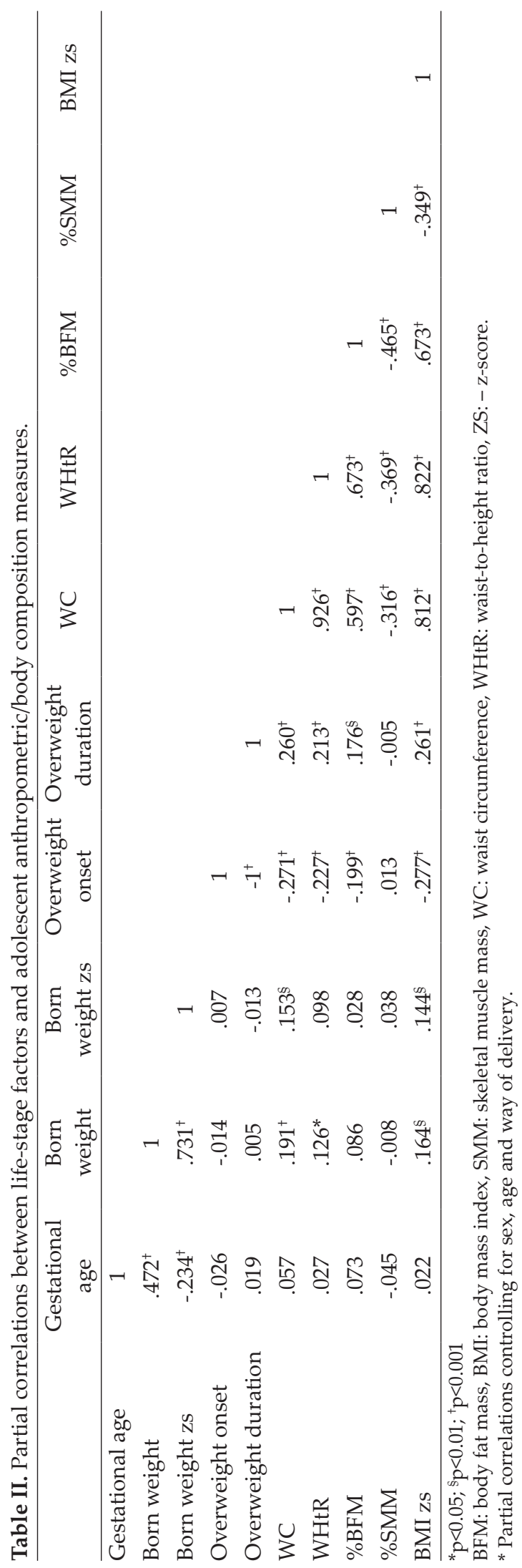

The Turkish Journal of Pediatrics • November-December 2020 
and later in life. ${ }^{25,26}$ According to our findings, CS was not associated with BMI z-score during adolescence. One possible explanation for the lack of association may be the specific characteristics of the sample studied. In fact, the majority of the studies that reported an association between CS and BMI z-score studied the general population. ${ }^{25}$ The absence in our sample of other weight categories, besides overweight, most probably impaired this association. Another possible explanation could be the adoption of healthy lifestyle behaviors at a certain point in time. Although the way of delivery may influence overweight onset, dietary and physical activity behaviors are concurrent and modifiable factors that may influence BMI z-score at any time. ${ }^{27}$ Indeed, in this study, CS showed to be only associated with overweight onset. Adolescents born by CS have shown decreased age (-1.4 \pm 0.47 years) of overweight onset, compared to other ways of delivery. According to the literature, the link between CS and increased BMI relies on the hygiene hypothesis. In other words, infants born by CS are mainly exposed to maternal skin microbiota and to external environmental bacterial communities at birth. CS impairs the exposure of the newborn to maternal vaginal bacteria, which is known to be the major source of the newborn's intestinal bacteria. The decreased intestinal bifidobacteria and bacteroids (known to be negatively associated with dietary nutrient absorption) in the newborn delivered by CS compared to vaginal, may lead to early overweight onset. Although data on intrapartum indications for CS is missing, it should be noted that the main intrapartum indications for this procedure are not known as risk factors for obesity..$^{28}$

In line with several other studies, our findings show a positive association between birth weight/birth weight z-score and BMI z-score, WC and WHtR. Interestingly, birth weight by itself showed higher association levels with BMI z-score, WC and WHtR during adolescence, compared to the birth weight $\mathrm{z}$-score. Although not further explored, other authors have reported similar results. ${ }^{6,729,30}$ In this study, birth weight was the second-best predictor of adolescent BMI z-score. In addition, gestational age was positively correlated with birth weight and negatively associated with birth weight z-score, with a higher correlation level within the former. These results suggest that the use of a z-score, adjusting for gestational age may have affected and biased the association between the variables under study. ${ }^{25} \mathrm{~A}$ similar finding regarding the use of birth weight $\mathrm{z}$-score was reported by Delbaere et al. ${ }^{31}$

Conversely to our hypothesis and to the results reported by other authors, ${ }^{5,32}$ overweight onset during the period of adiposity rebound was not associated with BMI and WC severity. Instead, overweight onset, as a continuum variable, was negatively correlated with BMI z-score, WC, WHtR and BFM. The negative correlation between overweight onset and WC/WHtR was as relevant as the one between overweight onset and BMI z-score. The association between WC and impaired metabolic profiles in adolescents with overweight has already been widely described. ${ }^{33,34}$

Regression analyses showed that overweight duration and not overweight onset was the best predictor of adolescent BMI z-score. Prolonged inadequate dietary and physical activity behaviors may lead to excessive weight gain and in turn, to harmful metabolic adaptations and adipose tissue dysfunction as early as in childhood. ${ }^{35}$ Adipose tissue dysfunction, is characterized by changes in adiponectin and leptin levels as well as in insulin sensitivity. ${ }^{35}$ However, it is not completely understood whether these metabolic changes are a consequence or a trigger of an adipose tissue increased proliferative capacity which may be potentiated by overweight duration. ${ }^{35}$ As overweight duration may lead to short and longrun psychological consequences, such as body dissatisfaction, low self-esteem, poor healthrelated quality of life and even depression it may negatively affect behavior change thus perpetuating the cycle. ${ }^{36,37}$ 
The retrospective nature of this study has not allowed for dietary and physical activity data collection. Another limitation is the crosssectional design, not allowing for causal inferences. Nevertheless, to the best of our knowledge, no other study has investigated the interaction between the method of delivery, birth weight and overweight onset on overweight severity in a sample of adolescents with overweight. The fact that this study brings further knowledge to the study of BMI severity during adolescence is its main strength.

In summary, this study showed a positive association between CS and early overweight onset, which reinforces the need for a careful assessment of the risk-benefit balance to conduct a CS on an individual basis. Overweight onset and particularly overweight duration were associated with BMI z-scores severity. Nevertheless, CS was not a perinatal predictor of adolescent BMI z-score, which suggests that life-style may interrupt the burden of CS on BMI z-score throughout growth. On the other hand, birth weight was the perinatal factor best associated with BMI z-score. This finding, in addition to the fact that overweight duration was found to be the best predictor of BMI z-score severity, led us to conjecture that increased birth weight may anticipate overweight onset, and consequently overweight duration in the presence of inadequate lifestyle behaviors.

\section{Funding}

AVS is supported by the Portuguese Foundation for Science and Technology (SFRH/ BD/130193/2017). AVS funding organization played no role in this research. This research did not receive any specific grant from funding agencies in the public, commercial, or not-forprofit sectors.

\section{REFERENCES}

1. Ng Marie, Fleming T, Robinson M, et al. Global, regional, and national prevalence of overweight and obesity in children and adults during 1980-2013: a systematic analysis for the Global Burden of Disease Study 2013. Lancet 2014; 384: 766-781.
2. Kelly T, Yang W, Chen CS, Reynolds K, He J. Global burden of obesity in 2005 and projections to 2030. Int J Obes (Lond) 2008; 32: 1431-1437.

3. Goldani HA, Bettiol H, Barbieri MA, et al. Cesarean delivery is associated with an increased risk of obesity in adulthood in a Brazilian birth cohort study. Am J Clin Nutr 2011; 93: 1344-1347.

4. Goldani MZ, Barbieri MA, da Silva AA, Gutierrez MR, Bettiol H, Goldani HA. Cesarean section and increased body mass index in school children: two cohort studies from distinct socioeconomic background areas in Brazil. Nutr J 2013; 12: 104.

5. Dietz WH. Periods of risk in childhood for the development of adult obesity-what do we need to learn? J Nutr 1997; 127: 1884S-1886S.

6. Araujo de Franca GV, De Lucia Rolfe E, Horta BL, et al. Associations of birth weight, linear growth and relative weight gain throughout life with abdominal fat depots in adulthood: the 1982 Pelotas (Brazil) birth cohort study. Int J Obes (Lond) 2016; 40: 14-21.

7. Araujo de Franca GV, Restrepo-Mendez MC, Loret de Mola C, Victora CG. Size at birth and abdominal adiposity in adults: a systematic review and metaanalysis. Obes Rev 2014; 15: 77-91.

8. Qiao Y, Ma J, Wang Y, et al; and for the ISCOLE Research Group. Birth weight and childhood obesity: a 12-country study. Int J Obes Suppl 2015; 5(Suppl 2): S74-S79.

9. Gillman MW, Rifas-Shiman S, Berkey CS, Field AE, Colditz GA. Maternal gestational diabetes, birth weight, and adolescent obesity. Pediatrics 2003; 111: e221-e226.

10. Reilly JJ, Armstrong J, Dorosty AR et al; Avon Longitudinal Study of Parents and Children Study Team. Early life risk factors for obesity in childhood: cohort study. BMJ 2005; 330: 1357.

11. Frisancho AR. Prenatal compared with parental origins of adolescent fatness. Am J Clin Nutr 2000; 72: 1186-1190.

12. Bettiol H, Sabbag Filho D, Haeffner LSB, et al. Do intrauterine growth restriction and overweight at primary school age increase the risk of elevated body mass index in young adults? Braz J Med Biol Res 2007; 40: 1237-1243.

13. Kang M, Yoo JE, Kim K, Choi S, Park SM. Associations between birth weight, obesity, fat mass and lean mass in Korean adolescents: the Fifth Korea National Health and Nutrition Examination Survey. BMJ Open 2018; 8: e018039.

14. Rolland-Cachera MF, Deheeger M, Maillot M, Bellisle F. Early adiposity rebound: causes and consequences for obesity in children and adults. Int J Obes (Lond) 2006; 30(Suppl 4): S11-S17. 
15. Williams SM, Goulding A. Patterns of growth associated with the timing of adiposity rebound. Obesity (Silver Spring) 2009; 17: 335-341.

16. Geserick M, Vogel M, Gausche R, et al. Acceleration of BMI in early childhood and risk of sustained obesity. N Engl J Med 2018; 379: 1303-1312.

17. Videira-Silva A, Sardinha L, Fonseca H. Effect of a physical activity consultation in the management of adolescent overweight (the PAC-MAnO project): study rationale, design and methods. BMJ Paediatr Open 2018; 2: e000214.

18. Inchley J, Currie D, Jewell J, Breda J, Barnekow V (eds). Adolescent obesity and related behaviors: trends and inequalities in the WHO European Region, 2002-2014. World Health Organization, 2017; 1-98.

19. Bassali R, Waller JL, Gower B, Allison J, Davis CL. Utility of waist circumference percentile for risk evaluation in obese children. Int J Pediatr Obes 2010; 5: 97-101.

20. Videira-Silva A, Fonseca H. The effect of a physical activity consultation on body mass index z-score of overweight adolescents: results from a pediatric outpatient obesity Clinic. Eur J Pediatr 2017; 176: 655-660.

21. The NS, Suchindran C, North KE, Popkin BM, Gordon-Larsen P. The association of adolescent obesity with risk of severe obesity in adulthood. JAMA 2010; 304: 2042-2047.

22. Chatfield A, Caglia JM, Dhillon S, et al; International Fetal and Newborn Growth Consortium forthe 21st Century. Translating research into practice: the introduction of the INTERGROWTH-21st package of clinical standards, tools and guidelines into policies, programmes and services. BJOG 2013; 120(Suppl 2): 139-142.

23. McTigue KM, Garrett JM, Popkin BM. The natural history of the development of obesity in a cohort of young U.S. adults between 1981 and 1998. Ann Intern Med 2002; 136: 857-864.

24. Sahoo K, Sahoo B, Choudhury AK, Sofi NY, Kumar R, Bhadoria AS. Childhood obesity: causes and consequences. J Family Med Prim Care 2015; 4: 187192.

25. Darmasseelane K, Hyde MJ, Santhakumaran S, Gale G, Modi N. Mode of delivery and offspring body mass index, overweight and obesity in adult life: a systematic review and meta-analysis. PLoS One 2014; 9: e87896.

26. Mesquita DN, Barbieri MA, Goldani HAS, et al. Cesarean section is associated with increased peripheral and central adiposity in young adulthood: cohort study. PLoS One 2013; 8: e66827.
27. Carlson JA, Crespo NC, Sallis JF, Patterson RE, Elder JP. Dietary-related and physical activity-related predictors of obesity in children: a 2-year prospective study. Child Obes 2012; 8: 110-115.

28. Barber EL, Lundsberg LS, Belanger K, Pettker CM, Funai EF, Illuzzi JL. Indications contributing to the increasing cesarean delivery rate. Obstet Gynecol 2011; 118: 29-38.

29. Matthews EK, Wei J, Cunningham SA. Relationship between prenatal growth, postnatal growth and childhood obesity: a review. Eur J Clin Nutr 2016; 71: 919-930.

30. Yu ZB, Han SP, Zhu GZ, et al. Birth weight and subsequent risk of obesity: a systematic review and meta-analysis. Obes Rev 2011; 12: 525-542.

31. Delbaere I, Vansteelandt S, De Bacquer D, et al. Should we adjust for gestational age when analysing birth weights? The use of z-scores revisited. Hum Reprod 2007; 22: 2080-2083.

32. De Kroon ML, Renders CM, Van Wouwe JP, Van Buuren S, Hirasing RA. The Terneuzen birth cohort: BMI changes between 2 and 6 years correlate strongest with adult overweight. PLoS One 2010; 5: e9155.

33. Hirschler V, Aranda C, Calcagno Mde L, Maccalini G, Jadzinsky M. Can waist circumference identify children with the metabolic syndrome? Arch Pediatr Adolesc Med 2005; 159: 740-744.

34. Freedman DS, Serdula MK, Srinivasan SR, Berenson GS. Relation of circumferences and skinfold thicknesses to lipid and insulin concentrations in children and adolescents: the Bogalusa Heart Study. Am J Clin Nutr 1999; 69: 308-317.

35. Landgraf K, Rockstroh D, Wagner IV, et al. Evidence of early alterations in adipose tissue biology and function and its association with obesity-related inflammation and insulin resistance in children. Diabetes 2015; 64: 1249-1261.

36. Neumark-Sztainer D, Story M, French SA, Hannan PJ, Resnick MD, Blum RW Psychosocial concerns and health- compromising behaviors among overweight and nonoverweight adolescents. Obes Res 1997; 5: 237-249.

37. Zeller MH, Modi AC. Predictors of health-related quality of life in obese youth. Obesity (Silver Spring) 2006; 14: 122-130. 